\title{
Essentials of Neurosurgical Anesthesia \& Critical Care: Strategies for Prevention, Early Detection, and Successful Management of Perioperative Complications
}

\author{
Ansgar M. Brambrink, Jeffrey R. Kirsch (Editors). Springer New York, Dordrecht, \\ Heidelberg, London, 2012, 913 pages. ISBN 978-0-387-09561-5
}

\author{
Ryan S. Smith, MD • Rosemary Ann Craen, MBBS
}

Received: 21 September 2012/Accepted: 4 October 2012/Published online: 8 November 2012

(C) Canadian Anesthesiologists' Society 2012

This clinical handbook provides a comprehensive overview of commonly encountered problems in the management of neurosurgical and neurocritical care patients. Edited by two well-respected clinicians in neuroanesthesia and neurocritical care from Oregon Health and Science University, more than 150 colleagues have contributed to this topical and complete text. The editors intend this handbook to be a quick reference guide for frontline providers, including physicians, trainees, physician assistants, and advanced practice nurses who care for neurosurgical patients in the perioperative period. The book aims to help providers anticipate and effectively manage perioperative neurosurgical complications.

Brambrink and Kirsch have edited a voluminous handbook. The book's 91 chapters are effectively organized into fourteen sections that encompass the management of neurosurgical patients from basic anatomy and physiology, preoperative assessment, and fundamentals of neuroanesthesia through intraoperative emergencies and critical situations for both adult and pediatric patients, and finally through management challenges in the postanesthesia and intensive care units. It is particularly helpful that specific sections and chapters are dedicated to specialized pediatric problems, recognizing that neurosurgical care in these patients certainly requires a different set of considerations and skills. Hot topics, such as neuromonitoring, brain protection, endovascular therapies, awake craniotomy,

R. S. Smith, MD - R. A. Craen, MBBS ( $)$

Schulich School of Medicine \& Dentistry, Western University, London, ON, Canada

e-mail: rosemary.craen@lhsc.on.ca endoscopic neurosurgery, brain death, and organ donation, are addressed and written by experts in the field.

The handbook format is an appropriate choice for this text given the breadth of topics that are included. The organization and flow are logical, each major topic section is covered fully, and the authors deal with each topic at an appropriate level of detail for a clinical handbook. Most chapters range from ten to fifteen $(8.5 \times 5.5 \mathrm{in})$ pages. Important points are addressed, but the reader is well advised by the editors to seek out other sources if a more comprehensive review is desired. The editors provide a list of suggested readings at the conclusion of each chapter to help the reader access further detail. The text contains both a complete and descriptive table of contents and a comprehensive and effective index. Tables are included sparingly but effectively display information that is referenced in the body of the text. Figures are inserted where appropriate, and without distraction, to reinforce the text, and colour is employed where required for clarity. The key points box at the end of each chapter allows for quick access to crucial information.

We centre our primary criticism of the book on referencing. The editors of this handbook chose not to cite references, which can be problematic for the reader who chooses to probe a statement in the text in order to obtain further detail or to assess the accuracy of the material. Furthermore, in several chapters, the works on the suggested reading list are more than five years old, e.g., in Chapter 18, the resources for hypothermia and neuroprotection date from 2005 to 2007. A humble suggestion to improve future editions would be to reference major points in the text and to include the most recent literature in the suggested reading lists. We do recognize the inherent time lag between researching, writing, and publishing the work. 
This book is unique as a clinical handbook that essentially covers the major topics of neuroanesthesia in their entirety, albeit at an overview level. Most other handbooks, while being smaller in volume, cover only a portion of the subspecialty. The editors have successfully achieved the stated goal of providing a comprehensive at-hand reference for frontline clinicians to manage both common and unusual perioperative neuroanesthesia complications. This book would be a valuable addition to the library of a clinician who requires the occasional high-level review or desires a starting point for in-depth learning. The chapters of this book could be used effectively as templates for structuring bedside teaching of trainees.
Overall, this is a well-written, well-edited, and wellorganized clinical handbook that covers the diversity of perioperative complications and emergencies in neuroanesthesia and critical care. Although we have pointed out the lack of references as a weakness, this is certainly outweighed by the impressive list of contributors who have drawn on decades of collective experience while creating this work. Essentials of Neurosurgical Anesthesia and Critical Care offers good value and would be a positive addition to the clinician's library.

Conflicts of interest None declared. 